\title{
A sintaxe e a semântica de expressões numéricas do Karitiana
}

\author{
The Syntax and Semantic of Karitiana \\ Numerical Expressions \\ Juliana Vignado Nascimento*
}

\begin{abstract}
Resumo
Este artigo analisa o sistema numérico da língua indígena brasileira Karitiana. O objetivo é explorar a estrutura sintática e semântica de numerais compostos, isto é, aqueles que derivam de numerais básicos e mecanismos sintáticos como coordenação e complementação. O sistema numérico Karitiana é composto por cinco numerais básicos e numerais compostos formados a partir dos numerais básicos e das palavras yjpy 'nossa mão', yjpi 'nosso pé, ota 'outro' e ot 'pegar'. Serão usados dois aportes teóricos para tratar os números compostos. A estrutura sintática será explicada através da estratégia de empacotamento de Hurford (1975, 1987, 2003, 2007), que postula que operações sintáticas expressam operações matemáticas como multiplicação e adição, necessárias para a formação de numerais compostos. A estrutura semântica, por sua vez, será explicada através da Semântica Fregeana para palavras numéricas, desenvolvida por Rothstein (2013) a partir de Chierchia (1985) e Frege (1982). O sistema numérico Karitiana é de base cinco, e os numerais compostos são formados via bases multiplicadoras e coordenação com a palavra ot. Até o momento, duas bases morfêmicas multiplicativas foram identificadas na língua: yjpy e yjpi, que, apesar do fato de valerem 5 cada, se distribuem de maneira complementar e hierarquizada. Hurford propõe que a estratégia de empacotamento é um fenômeno universal nas línguas naturais para a formação de números compostos. Este artigo mostra que essa estratégia também se aplica a uma língua tipologicamente diferente, como o Karitiana, o que corrobora a proposta de que esse seja um fenômeno universal.
\end{abstract}

Palavras-chaves: Numeral, Lingua indígena, Estratégia de Empacotamento

* Universidade de São Paulo 


\section{Abstract}

This paper analyzes the numerical system of the Brazilian indigenous language Karitiana. The aim of this study is to explore the syntactic and semantic structure of complex numerals, that is, those derived from basic numerals and syntactic mechanisms such as coordination and complementation. The Karitiana numerical system consists of five basic numerals, and complex numerals formed from the basic numerals and the words yjpy 'our hand', yjpi 'our foot' ota 'other' and ot 'catch'. Two theoretical contributions will be used to treat the complex numbers. The syntactic structure will be explained through the Hurford's packaging strategy $(1975,1987,2003,2007)$, which postulates that syntactic operations express mathematical operations such as multiplication and addition, necessary for the formation of compound numerals. The semantic theory used is the Fregean semantics for numerical words developed by Rothstein (2013) from Chierchia (1985) and Frege (1982). Karitiana numerical system is base five, and the complex numerals are formed via multiplying bases and coordination with the word ot. Until now, two multiplicative morphemic bases have been identified in this language: yjpy and yjpi, which, despite the fact that they are worth 5 each, are distributed in a complementary and hierarchical way. It can be concluded that the packing strategy operates on the Karitiana numerals, which enhances the universality of this mechanism in natural languages.

Keywords: Numeral, Indigenous language, Packing Strategy

Revista Letras, 


\section{Introdução ${ }^{1}$}

548

importância do estudo de línguas indígenas brasileiras está no fato
de serem bastante relevantes para as teorias linguísticas por suas - 1 singularidades, além de serem ainda pouco conhecidas, pouco descritas, e de muitas delas estarem ameaçadas de extinção. É comum encontrar línguas da Amazônia que têm substituído ou suplementado os seus sistemas numéricos nativos por empréstimos do Português ou do Espanhol. Assim, sistemas numéricos constituem um dos traços mais ameaçados, não somente das línguas da Amazônia, como também em demais línguas minoritárias (EPPS; SALANOVA, 2012).

Comrie (2005) observou que a ameaça aos sistemas numéricos nativos é reforçada pela vasta difusão do sistema numérico decimal através das línguas do mundo, sendo um dos principais fatores dessa difusão a educação formal e o mercado financeiro. Fargetti (2015) destaca que sistemas numéricos ainda são uma lacuna nos estudos linguísticos no Brasil. Além disso, a necessidade de descrições de línguas indígenas é reforçada pelo fato de que para apenas 83 das cerca de 160 línguas indígenas atualmente faladas no Brasil existe algum tipo de descrição (LEITE e FRANCHETTO, 2001).

1 Agradecimentos ao $\mathrm{CNPq}$ pelo apoio financeiro que possibilitou a produção deste artigo. 
J. N.

VignADO

A sintaxe e

a semântica

de expressões

numéricas no

Karitiana
Revista Letras,

Curitiba, UfPr, n. 96, pp.546-565,

jul./dez. 2017 ISSN 2236-0999 (versão eletrônica)
Os sistemas numéricos variam nas línguas naturais em relação a sua base numérica, em termos matemáticos, e, em relação a sua realização linguística, em termos morfológicos. Esses sistemas são formados por uma classe de numerais cardinais básicos, que são: um, dois, três, quatro, cinco, seis, sete, nove e dez em Português, por exemplo, e uma classe de numerais compostos que são formados via os numerais básicos e outros mecanismos morfossintáticos como sufixação e coordenação, como por exemplo os números dezessete e duzentos e trinta e dois em Português.

Sistemas numéricos são recursivos assim como a linguagem humana, já que a partir de unidades mínimas pode-se produzir infinitos numerais. A essa capacidade de construir expressões numéricas, chama-se faculdade numérica (CORVER et al, 2007). Chomsky (1998) sugere que a faculdade numérica é uma abstração da faculdade da linguagem, pois mantém a infinitude segmentada, que corresponde à capacidade de compor infinitas expressões a partir de um conjunto finito e segmentado de elementos.

Nessa perspectiva, partindo de uma abordagem linguística gerativa, este artigo pretende explorar a existência de mecanismos linguísticos universais na formação sintática de numerais. Para isso, o objetivo principal é analisar a sintaxe dos numerais do sistema numérico Karitiana usando a teoria da estratégia de empacotamento desenvolvida por Hurford $(1975,1987,2007)$ e estabelecer uma interface com a semântica a partir da semântica de tipos de Rothstein (2013).

Os dados do sistema numérico apresentados neste artigo foram coletados de duas maneiras. Em um primeiro momento, os numerais Karitiana foram coletados a partir da literatura científica sobre a língua (ver LANDIN, 1984, 2005; MULLER et al, 2006). Depois, em outubro de 2015, foram coletados dados de numerais com dois informantes nativos. Um dos objetivos dessa coleta foi subsidiar um trabalho de conferência dos numerais que já haviam sido listados anteriormente, com o objetivo de garantir a qualidade dos dados (VIGNADO, 2016) $)^{2}$.

Esta análise parte da hipótese de que expressões numéricas devem ser avaliadas como construções sintáticas, ao invés de apenas compostos morfológicos. Dentro dessa perspectiva, a análise dos dados mostra que a Estratégia de Empacotamento opera na formação de numerais compostos na língua Karitiana e estabelece uma interface semântica baseada na noção de tipos semânticos. A análise da recursividade numérica e morfológica dos numerais mostrou que esse é um sistema de base 5, diferente do que foi descrito anteriormente por Green (1997), que classificou o sistema numérico Karitiana como um sistema de base 20.

O presente artigo está organizado como segue: a seção 1 apresenta brevemente a língua Karitiana e contém informações básicas do seu sistema numérico. $\mathrm{Na}$ seção 2 é apresentado o panorama teórico aplicado neste

2 Esse trabalho foi realizado no quadro de uma iniciação científica realizada pela autora deste artigo com o apoio do CNPq em 2015-2016. 
artigo e contém duas subseções, 2.1, que apresenta a teoria da Estratégia de Empacotamento de Hurford, e 2.2, que apresenta a teoria de tipos semânticos de Rothstein. Na seção 3 são apresentados a análise e os resultados, e a seção 4 traz as considerações finais.

\section{O Karitiana e seu sistema numérico}

Esta seção traz informações sobre a língua Karitiana, a descrição de seu sistema numérico na literatura tipológica e uma tabela que apresenta seus numerais e sua decomposição morfológica.

O Karitiana é uma língua indígena brasileira cuja tribo homônima vive em uma reserva localizada próxima à cidade de Porto Velho, capital do estado de Rondônia. Essa etnia abrange cerca de 400 indivíduos e conserva o uso de sua língua. A língua Karitiana pertence à família Tupi e à sub-família Arikém. Desta sub-família, Rodrigues (1986) mostra que foram identificadas outras duas línguas extintas, o Kabixiana e o Arikém, sendo assim o Karitiana sua única língua sobrevivente.

Green (1997) descreve 47 sistemas numéricos de línguas indígenas. Essa autora diz que as línguas cujo sistema numérico é de base vigesimal têm palavras distintas para os numerais de 1 a 5 ; o termo para 5 significa "nossa mão", "todos os dedos da mão" ou “o fim da mão"; e daí em diante são usados a outra mão e os pés para expressar os numerais maiores. Para essa autora, alguns exemplos de línguas que utilizam esse sistema são: Karajá (Macro-Jê (ALFORD,1987); (KARAJÁ; FORTUNE; FORTUNE, 1989)), Rikbaktsá (Macro-Jê (BOSWOOD, 1978)), Urubú-Kaapor (Tupi-Guaraní (KAKUMASU; KAKUMASU, 1988)), Kadiwéu (Guaikurú (GRIFFITHS, 1975)), Karitiana (Arikém (LANDIN, 1983)), Tikúna (isolada (ANDERSON, 1958)), Makuxí (Karib (WILLIAMS, 1932)), etc.

O caráter tipológico e quantitativo desse estudo de Green, entretanto, não contemplou a análise sintática dos termos numerais de forma mais profunda, e inclui algumas notas de rodapé sobre exceções à descrição geral que ela faz desses sistemas. A autora afirma:

\footnotetext{
Os sistemas vigesimais dessas línguas são quinários, pois os numerais maiores do que cinco compõem-se dos mesmos cinco termos, e o numeral 10 é considerado uma combinação de 'cinco mais cinco' (mão mais mão). (GREEN, 1997, p. 11)
}

O Karitiana possui cinco numerais básicos e mais numerais compostos, porém, os numerais básicos ainda são utilizados pela maioria dos falantes em seu cotidiano, enquanto os numerais compostos estão sendo esquecidos. Para expressar quantidades maiores, os falantes optam pelos numerais do Português. Esse fato é bastante comum em línguas minoritárias, que acabam realizando 
J. N. empréstimos dos numerais das línguas oficiais dos países em que estão inseridas

VIGNADO

A sintaxe e

a semântica

de expressões

numéricas no

Karitiana pelo contato da população com o comércio e a educação formal.

Os numerais básicos nessa língua sempre aparecem com o sufixo $\{-t\}$, que, em contexto nasal, é realizado como /p/. Muller et al (2006) atentam para o fato de que o único contexto em que esse morfema não aparece é em repetição, em que se usa o sufixo enfático\{-o\}. Por exemplo, o número 'dois' sypomp, quando é resposta para uma pergunta como $O$ que você disse?, recebe o sufixo enfático e se torna sypomo. Em todos os demais contextos esse sufixo aparece ligado ao numeral.

Storto $(1999,2003)$ descreve o sufixo $\{-t\}$ como um sufixo oblíquo e de natureza posposicional. Assim, o numeral formaria com o sufixo $\{-t\}$ um sintagma posposicional de contagem. Contudo, foi verificada a existência de um sufixo adverbializador $\{-t\}$ em quantificadores adverbiais como pitat 'muito' (SANCHEZ-MENDES, 2009) e outros elementos adverbiais como asoasot 'rapidamente' (VIVANCO, 2014). Esse fato reforça a hipótese de que o sufixo presente nos numerais é um adverbializador, já que é mais econômico assumir um sufixo adverbializador do que assumir que a língua apresenta a mesma forma fonológica para sufixos com funções diferentes (VIGNADO, 2016).

Muller et al (2006) demonstram que não há material funcional no sintagma nominal, nem tampouco quantificação, marcação de plural, marcação de definitude ou de gênero. Portanto, o Karitiana é uma língua que concentra suas informações funcionais no sintagma verbal. Esses autores também demonstraram que, em Karitiana, os nomes são cumulativos, se um animal pikom ('macaco') é somado a outro animal pikom, dizemos que os dois animais são pikom, ou seja, o nome denota tanto entidades atômicas quanto plurais.

As posições sintáticas dos adverbiais na língua em sentenças matrizes são AdvSVO, SVAdvO, SVOAdv e *SAdvVO (ver STORTO, 1999). A sentença abaixo, em (1), e suas variações em a),b) e c) ilustram essas possibilidades, note que o significado não se altera.

$\begin{array}{llcll}\text { (1) a. } & \text { mynda } & \text { taso } & \text { nampotporaj } & \text { ese } \\ & \text { mynda } & \text { taso } & \text { na-m-potpora-j } & \text { ese } \\ & \text { vagarosamente } & \text { homem } & \text { DECL-CAU-fervert-FUT água } \\ & \text { taso nampotporaj mynda esse } & \\ \text { b. } & \text { taso nampotporaj ese mynda } & \\ \text { c. } & \text { *taso mynda nampotporaj ese } & \\ & \text { "homem(ns) ferverá(rão) água vagarosamente" }\end{array}$

(STORTO, 1999, p.139) 
Os numerais respeitam as mesmas restrições sintáticas que os demais elementos adverbiais na língua, como mostra o conjunto de sentenças em (2), abaixo:

$\begin{array}{lllll}\text { Jõnso } & \text { nakam'at } & \text { ep } & \text { ombaky } & \text { sypomp } \\ \text { Jõnso } & \text { naka-m-'at } & \text { ep } & \text { ombaky } & \text { sypom-t } \\ \text { Jõnso } & \text { DECL-CAUS-fazer madeira } & \text { onça } & \text { dois-ADV }\end{array}$

a. jõnso nakam'at sypomp ep ombaky

b. sypomp jõnso nakam'at ep ombaky

c. jõnso sypomp nakam’at ep ombaky 'mulher(es) fez(fizeram) duas onças de madeira'

(Informante C, outubro, 2015)

O sentido da sentença não é alterado com a mudança de posição do numeral, do mesmo modo que ocorre com demais adverbiais. Assim, levando-se em conta as posições sintáticas dos numerais, que respeitam as mesmas restrições dos demais adverbiais da língua, os numerais dessa língua devem ser elementos adverbiais. Na tradução da sentença nota-se que o sintagma nominal jõnso 'mulher' pode ser singular ou plural. Em Karitiana, os nominais são cumulativos, ou seja, denotam tanto entidades plurais quanto entidades atômicas. Se o numeral estivesse modificando o sintagma nominal jõnso em algumas dessas condições sintáticas, seria necessário que as condições de verdade da sentença garantissem a existência de duas mulheres, e assim, uma denotação necessariamente plural, o que não acontece (VIGNADO, 2016).

552 Apesar de não ser o escopo deste artigo, essa breve explicação justifica porque, nesta análise, o sufixo em questão será glosado como um adverbializador. Abaixo, a tabela (1) apresenta o sistema numérico Karitiana:

\begin{tabular}{|l|l|}
\hline Numeral & Decomposição morfológica GLOSAS:ADVZ-adverbializador 1p-primeira pessoadedural \\
\hline Um & $\begin{array}{l}\text { myhin-t } \\
\text { um-ADVZ }\end{array}$ \\
\hline Dois & $\begin{array}{l}\text { sypom-p } \\
\text { dois- ADVZ }\end{array}$ \\
\hline Três & $\begin{array}{l}\text { mỹjym-p } \\
\text { três-ADVZ }\end{array}$ \\
\hline Quatro & $\begin{array}{l}\text { otadnamyn-t } \\
\text { quatro-ADVZ }\end{array}$ \\
\hline Cinco & $\begin{array}{l}\text { yjpy-t } \\
\text { cinco-ADVZ }\end{array}$ \\
\hline Seis & $\begin{array}{l}\text { myhin-t yj-py ota ot } \\
\text { um-ADVZ 1p-mão outro pegar }\end{array}$ \\
\hline Sete & $\begin{array}{l}\text { sypom-p yj-py ota ot } \\
\text { dois-ADVZ 1p-mão outro pegar }\end{array}$ \\
\hline
\end{tabular}




\begin{tabular}{|c|c|c|}
\hline \multirow{13}{*}{$\begin{array}{r}\text { J. N. } \\
\text { Vignado } \\
\text { A sintaxe e } \\
\text { a semântica } \\
\text { de expressões } \\
\text { numéricas no } \\
\text { Karitiana }\end{array}$} & Oito & $\begin{array}{llc}\text { mỹjym-p } & \text { yj-py ota } & \text { ot } \\
\text { três-ADVZ } & \text { 1p-mão outro } & \text { pegar } \\
\end{array}$ \\
\hline & Nove & $\begin{array}{lccc}\text { otadnamyn-t } & \text { yj-py } & \text { ota } & \text { ot } \\
\text { quatro- ADVZ } & 1 \mathrm{p}-\text {-mão } & \text { outro } & \text { pegar } \\
\end{array}$ \\
\hline & Dez & $\begin{array}{lll}\text { yjpy } & \text { ota } & \text { tyy-tap } \\
\text { 1p-mão } & \text { outro } & \text { grande-(?) } \\
\end{array}$ \\
\hline & Onze & $\begin{array}{lcc}\text { myhin-t } & \text { yj-pi } & \text { ot } \\
\text { um-ADVZ } & 1 \text { p-pé } & \text { pegar } \\
\end{array}$ \\
\hline & Doze & $\begin{array}{lcc}\text { sypom-p } & \text { yj-pi } & \text { ot } \\
\text { dois-ADVZ } & 1 \text { p-pé } & \text { pegar } \\
\end{array}$ \\
\hline & Treze & $\begin{array}{lcc}\text { mỹjym-p } & \text { yj-pi } & \text { ot } \\
\text { três-ADVZ } & \text { 1p-pé } & \text { pegar } \\
\end{array}$ \\
\hline & Quatorze & $\begin{array}{lll}\text { otadnamyn-t } & \text { yj-pi } & \text { ot } \\
\text { quatro-t } & \text { 1p-pé } & \text { pegar } \\
\end{array}$ \\
\hline & Quinze & $\begin{array}{llc}\text { myhin-t } & \text { yj-pi } & \text { py } \\
\text { um-ADVZ } & \text { 1p-pé } & \text { mão } \\
\end{array}$ \\
\hline & Dezesseis & $\begin{array}{llc}\text { myhin-t } & \text { yj-pi } & \text { ota ot } \\
\text { um-ADVZ } & \text { 1p-pé } & \text { outro pegar } \\
\end{array}$ \\
\hline & Dezessete & $\begin{array}{llc}\text { sypom-p } & \text { yj-pi } & \text { ota ot } \\
\text { dois-ADVZ } & \text { 1p-pé } & \text { outro pegar } \\
\end{array}$ \\
\hline & Dezoito & $\begin{array}{lccc}\text { mỹjym-p } & \text { yj-pi } & \text { ota } & \text { ot } \\
\text { três-ADVZ } & 1 \text { p-pé } & \text { outro } & \text { pegar } \\
\end{array}$ \\
\hline & Dezenove & $\begin{array}{lcc}\text { otadnamyn-t } & \text { yj-pi ota ot } \\
\text { quatro-ADVZ } & 1 \text { p-pé } & \text { outro pegar } \\
\end{array}$ \\
\hline & Vinte & $\begin{array}{l}\text { yj-pi } \quad \text { ota } \quad \text { tyy-ta-p } \\
1 \text { p-pé }\end{array}$ \\
\hline
\end{tabular}

Tabela 1 - Sistema numérico Karitiana

A maioria dos numerais compostos são formados pelas palavras yjpy 'nossa mão', yjpi 'nosso pé', ot 'pegar' e ota 'outro'. Em contrapartida, os números dez, quinze e vinte não apresentam uma regularidade tão clara. Os números para dez e vinte apresentam o termo ty $y$ tap, que contém a palavra ty $y$ 'grande' e tap, cujo significado isolado não foi identificado. Processos de composição por justaposição em numerais são encontrados em outras línguas, como, por exemplo, dezessete (dez+sete) em Português ou tretuno (trenta+uno) em Italiano. Assim, no nível morfológico, no Karitiana parece ocorrer o mesmo, já que, apesar da estrutura interna dessas palavras, a construção numérica constitui uma palavra em termos de expressar em um sentido uno e não divisível a cardinalidade da expressão. 


\section{Formação de numerais compostos}

\subsection{Estratégia de empacotamento}

Esta seção apresenta a teoria da Estratégia de Empacotamento desenvolvida por Hurford (1975, 1987). A Estratégia de Empacotamento é uma restrição universal que atua em construções numéricas. É amplamente observada em sistemas numéricos que expressam multiplicação e adição via estruturas sintáticas (ver HURFORD, 1987; AMAECHI, 2014; KOŞANER, 2016). Trata-se de um mecanismo linguístico recursivo que constrói "pacotes" a partir da maior unidade básica do conjunto lexical de palavras numéricas da língua. Assim, operações sintáticas expressam operações matemáticas, como a adição e a multiplicação, que são necessárias para a formação recursiva de números. Hurford (2003) postula que todas as línguas com sistemas numéricos expandidos usam a adição e a multiplicação como operações semânticas principais na construção dos numerais compostos, e que as línguas podem usar partículas que marquem a operação de adição, e também que marquem a operação de multiplicação, mas esses casos são menos comuns.

Os princípios que regem a Estratégia de Empacotamento são: i) vá o mais longe que puder com os recursos que tem; ii) minimize a quantia de entidades com que lida. A operação da Estratégia de Empacotamento depende dos recursos lexicais disponíveis na língua, o que explica a grande variação nas bases morfológicas utilizadas pelas línguas naturais para expressar a lógica numérica subjacente ao seu sistema numeral.

O autor distingue duas categorias básicas na formação dos numerais: i) DIGIT é a categoria lexical de numerais básicos da língua; ii) M (MULTIPLIER) é a categoria de base morfêmica multiplicativa, que corresponde a um morfema que representa um valor cardinal fixo. Há, também, duas regras básicas para a formação dos numerais, que são apresentadas abaixo como apresentadas por Hurford (1987):

$$
\begin{aligned}
& \text { NUMBER } \longrightarrow\left\{\begin{array}{l}
\text { DIGIT } \\
\text { PHRASE (NUMBER }
\end{array}\right\} \\
& \text { PHRASE } \longrightarrow \quad \begin{array}{l}
\text { Interpretado como adição } \\
\text { (NUMBER) } M
\end{array} \quad \text { Interpretado como multiplicação }
\end{aligned}
$$

Nesta análise, optou-se por manter os nomes dos constituintes em inglês para evitar ruídos no uso dos conceitos. NUMBER são os numerais do ponto de vista lexical, que podem ser formados por um DIGIT, por exemplo dois, ou 
J. N. pela adição de um PHRASE e um NUMBER, por exemplo quarenta e quatro.

VIGNADO

A sintaxe e

a semântica

de expressões

numéricas no

Karitiana PHRASE, por outro lado, é a estrutura interpretada como multiplicação, em que um NUMBER é o complemento de um M. PHRASE, contudo, também pode ser usado como um NUMBER. A tabela (2) abaixo resume as regras da estratégia de empacotamento com exemplos do Português:

\begin{tabular}{|l|l|}
\hline Regras & Exemplos $^{2}$ \\
\hline $\mathbf{M} \rightarrow$ Base morfêmica multiplicativa & $\{$-cento $\}$ \\
\hline $\begin{array}{l}\text { NUMBER } \rightarrow \text { DIGIT } \\
\text { PHRASE }\end{array}$ & $\begin{array}{l}\text { três } \\
\text { trinta }\left(3^{\star} 10\right)\end{array}$ \\
\hline $\begin{array}{l}\text { NUMBER } \rightarrow \text { PHRASE + DIGIT } \\
\text { (interpretado como adição) }\end{array}$ & $\left(\{\text {-cento }\}^{\star}\right.$ dois $)+$ três $=$ duzentos e três \\
\hline $\begin{array}{l}\text { PHRASE } \rightarrow \text { M x NUMBER } \\
\text { (interpretado como multiplicação) }\end{array}$ & $\{\text {-cento }\}^{\star}$ dois $=$ duzentos \\
\hline
\end{tabular}

Tabela 2 - Regras da estratégia de empacotamento

Por causa da estratégia de empacotamento, em qualquer parte de uma estrutura numérica, o constituinte irmão de um NUMBER deve ter o maior valor possível, isto é, deve ter uma cardinalidade maior do que a cardinalidade do NUMBER. Esse mecanismo é responsável pela distribuição das bases morfêmicas multiplicativas de um sistema, e essas bases são organizadas em uma ordem definida no sistema numérico; é essa ordenação que confere o valor cardinal de cada base. Esse mecanismo também garante que exista apenas uma expressão bem formada para cada número, e impede, por exemplo, a formação de expressões como * duzentos cem.

Abaixo, são ilustradas as estruturas sintáticas dos números duzentos e quarenta e dois em (3) e setecentos mil em (4) do Português a partir dessa teoria:

(3)

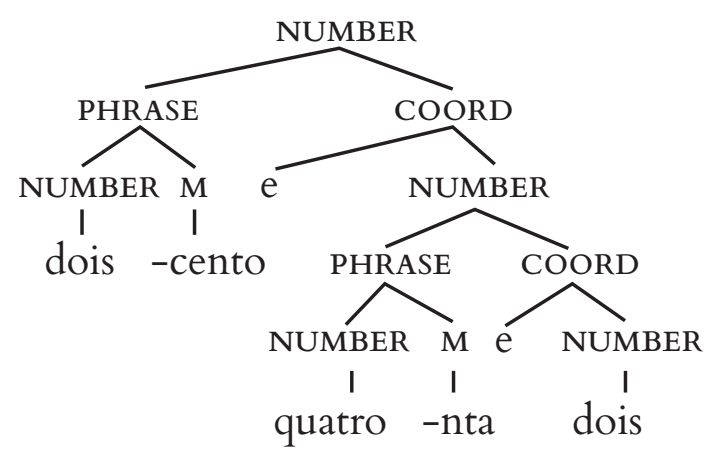

(4)

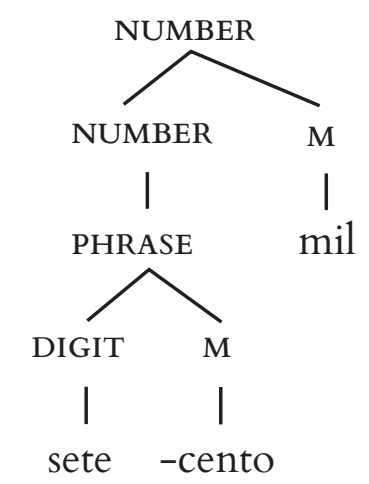

Revista Letras, 
No Português, a estrutura de adição é possibilitada pelo uso de uma conjunção coordenativa aditiva, o que é muito comum nas línguas naturais. Ionin e Matushansky (2006) demonstraram que os marcadores da operação aritmética de adição são frequentemente conjunções e adposições, como em Inglês and, em Francês et, em Hausa $d a$, em Igbo na, como mostrado no conjunto de dados (5)-(8), abaixo:

\begin{tabular}{|c|c|c|c|c|}
\hline Inglês & (5) & Five hund & and & eight $=508$ \\
\hline & & cinco cem & $e$ & oito \\
\hline Francês & (6) & Vingt- & et- & un $=21$ \\
\hline & & vinte & $e$ & $u m$ \\
\hline Hausa & (7) & Ashrin & $\mathrm{da}$ & bakwai $=27$ \\
\hline & & vinte & $e$ & sete \\
\hline Igbo & (8) & Iri & na & ise $=35$ \\
\hline & & dez & $e$ & cinco \\
\hline
\end{tabular}

O uso de conjunções pode ser observado nos exemplos, e usualmente não há marcação para a operação aritmética de multiplicação. Assim, na maioria das línguas, a ausência de uma marca morfológica entre dois elementos dentro de uma expressão numérica indica uma multiplicação.

\subsection{Tipos semânticos das palavras numéricas}

Esta seção apresenta a teoria semântica utilizada neste artigo. A interface semântica na formação de numerais compostos foi realizada a partir da semântica Fregeana para palavras numéricas apresentada por Rothstein (2013). A autora assume que (i) cardinais são sensíveis à distinção massivo e contável, (ii) a cardinalidade é uma propriedade de coisas contáveis e (iii) os numerais do inglês são modificadores cardinais adjetivais.

Para conferir uma análise semântica de numerais que trate dos dois modos de uso de um numeral, como um predicado e como um argumento, Rothstein adota, e adapta, a Property Theory de Chierchia (1985), que, por sua vez, segue Frege (1982), ao assumir que propriedades têm dois modos de interpretação: (i) não saturada, onde operam como predicados $\langle e, t\rangle$, como azul em "o mar é azul" e (ii) saturada, em que operam como argumentos $\langle\mathrm{e}\rangle$, como azul em "azul é minha cor favorita”.

Assim, para Rothstein, os numerais operam como propriedades. E como as demais propriedades de uma língua, têm um uso insaturado como predicado e um uso saturado como argumento. Como predicado, o numeral é de tipo $<e, t>$, mas como um argumento é de tipo <n>, o tipo semântico de numerais. 
J. N. O exemplo (9) abaixo mostra os numerais de tipos semânticos diferentes e suas

Vignado

$A$ sintaxe e

a semântica

de expressões

numéricas no

Karitiana denotações:

$$
\begin{aligned}
& {[[\text { quatro }<\mathrm{n}>]]=\lambda \mathrm{n} .|\ln |=4} \\
& {[[\text { quatro }<\mathrm{e}, \mathrm{t}>]]=\lambda \mathrm{x} .|\mathrm{x}|=4}
\end{aligned}
$$

$\mathrm{Na}$ teoria sintática de Hurford, as estruturas interpretadas como multiplicação são formadas com elementos que ele chama de Base Morfêmica Multiplicativa. A correspondência a esses elementos no modelo de Rothstein é o que ela chama de Léxico Multiplicador, cujo tipo semântico é $\langle\mathrm{n}\langle\mathrm{e}, \mathrm{t}\rangle\rangle$, no qual toma um numeral de tipo $<\mathrm{n}>$ e devolve um predicado de tipo $<\mathrm{e}, \mathrm{t}\rangle$ que contém uma operação de multiplicação, como o exemplo (10):

$$
\left[\left[\text { cento }_{\langle\mathrm{n}<e, t\rangle\rangle}\right]\right]=\lambda \mathrm{n} \lambda \mathrm{x} .|\mathrm{x}|=100^{\star} \mathrm{n}
$$

A autora apresenta também a denotação da conjunção coordenativa, que toma um elemento de um tipo $<\alpha>$ qualquer, e devolve um elemento de tipo $<\alpha$, $\alpha>$ que contém uma operação de soma. $\mathrm{O}$ exemplo (11) apresenta um "e" para elementos de tipo $\langle$ n $>$ e um "e" para elementos de tipo $\langle\mathrm{e}, \mathrm{t}>$ :

$$
\begin{aligned}
& {[[\mathrm{e}<\mathrm{n}<\mathrm{n}, \mathrm{n}>>]]=\lambda \mathrm{m} \lambda \mathrm{n} . \mathrm{n}+\mathrm{m}} \\
& {[[\mathrm{e}<\mathrm{et}<\mathrm{et}, \mathrm{et}>>]]=\lambda \mathrm{P} \lambda \mathrm{Q} . \mathrm{P}+\mathrm{Q}}
\end{aligned}
$$

A coordenação em uma expressão numérica expressa a operação de adição, e, assim, é definida como uma relação de soma entre os elementos envolvidos. $\mathrm{Na}$ formação de numerais, a coordenação toma dois numerais $n$ e $m$ e soma as suas cardinalidades.

\section{Análises e resultados}

Os dados do Karitiana mostram uma regularidade no uso das palavras yjpy e yjpi. A tabela (3) abaixo apresenta o paradigma dos números \{dois, sete, doze, dezessete\} para ilustrar a regularidade na formação desses numerais. $\mathrm{Na}$ primeira linha estão os numerais, na segunda linha, a referência da expressão, e, na terceira, a lógica matemática subjacente à expressão numérica. 


\begin{tabular}{|c|c|c|c|c|}
\hline Numeral & Sypomp (2) & $\begin{array}{l}\text { Sypomp yjpy } \\
\text { ota ot }(7)\end{array}$ & $\begin{array}{l}\text { sypomp yjpi } \\
\text { ot (12) }\end{array}$ & $\begin{array}{l}\text { sypomp yjpi ota ote } \\
\text { (17) }\end{array}$ \\
\hline Referência & II & ll & ஜ & \\
\hline $\begin{array}{l}\text { Composição } \\
\text { Arittética }\end{array}$ & $2+\left(0^{\star} 5\right)$ & $2+\left(1^{\star} 5\right)$ & $2+\left(2^{\star} 5\right)$ & $2+\left(3^{\star} 5\right)$ \\
\hline
\end{tabular}

Tabela 3 - Paradigma dos números \{dois, sete, doze, dezessete\}

Os números nesse paradigma aumentam de cinco em cinco no nível matemático, enquanto a expressão linguística mostra que o número é indicado pelo membro do corpo a que se refere. Assim, por exemplo, no número "doze" sypomp yjpi ot, ao invés de usar yjpy "nossa mão", a língua usa yjpi “nosso pé”. A tradução dessa expressão é 'pegue dois do pé', o que significa que as duas mãos, que somam dez, já foram contadas.

De acordo com Comrie (2005), a base aritmética de um sistema numérico é o valor $n$ sobre o qual as expressões numéricas se constroem seguindo um padrão, e é sobre esse valor $n$ que os multiplicadores M são baseados. A lógica da recursividade numérica é expressa pela fórmula $x . n+\gamma$, ou seja, algum numeral $x$ multiplicado pela base $n$ e somado a um numeral $\gamma$. De acordo com a exposição feita na Tabela 2, a base aritmética do sistema Karitiana é 5. Green (1997) destaca que os sistemas de base 20 são quinários, o que torna opaca a noção de base numérica que a autora considera. A análise proposta neste artigo mostra que a recursividade das bases morfêmicas expressam uma recursividade numérica de base 5 , e não de base 20 . Além do mais, esse sistema numérico é restrito e não apresenta regularidade nos números maiores que 20 .

As bases morfêmicas multiplicativas dessa língua são expressas linguisticamente por partes do corpo que, apesar de valerem 5 cada, são distribuídas de forma fixa e hierárquica nas seguintes estruturas: yjpy ota ot "pegue a outra mão"> yjpi ot "pegue um pé» > yjpi ota ot "pegue o outro pé". Essa distribuição das bases está de acordo com a regra da estratégia de empacotamento de que para qualquer parte de uma estrutura numérica, o irmão de um NUMBER deve ter o maior valor possível. Se não fosse esse o caso, seria possível fazer referência a valores maiores que dez tanto por meio de yjpy quanto de yjpi, mas o uso da base yjpi está de acordo com essa distribuição porque ela é a maior base.

Hurford (2007) aponta que a estratégia de empacotamento depende do léxico disponível na língua. Nos numerais Karitiana as palavras ota "outro", ot "pegar" e tyytap "todos” são necessárias na formação dos numerais compostos. A expressão linguística dos numerais maiores que dez usa a base yjpi "nosso pé”, que pressupõe que as mãos já foram contadas, e, para expressar números maiores que quinze, conta-se o outro pé. Por exemplo, no esquema anterior da tabela (3) pode-se observar, nos números sypomp yjpy ota ot "sete" e sypomp 
J. N.

VIGNADO

A sintaxe $e$

a semântica

de expressões

numéricas no

Karitiana

yjpi ota ot "dezessete", que a palavra ota é usada sempre que o outro membro é contado. Se essa palavra está associada às bases morfêmicas yjpy e yjpi, ela deve operar sintaticamente como um NUMBER. Na tabela (4) foram organizadas as estruturas multiplicativas que encontramos no Karitiana, cuja estrutura é composta por uma base morfêmica multiplicativa M e um NUMBER.

\begin{tabular}{|l|l|l|l|}
\hline$M($ number) & significado & valor & M x n \\
\hline yjpy (ota) & pegue outra mão & 5 & $5^{\star} 1$ \\
\hline yjpi & pé & 10 & $5^{\star} 2$ \\
\hline yjpi (ota) & pegue outro pé & 15 & $5^{\star} 3$ \\
\hline
\end{tabular}

Tabela 4 - Estrutura multiplicativas no Karitiana

É esperado que um NUMBER seja um numeral, numa relação de multiplicação com um MULTIPLIER (M). Isso é, portanto, um problema no caso do Karitiana, já que ota não parece ter um valor cardinal fixo, como os numerais tem. A palavra ota indica outro membro, por exemplo, yjpi ota significa o "outro pé". Usar a base yjpi significa que as mãos já foram contadas, e como são duas mãos, essa base vale $5^{\star} 2$. Já no caso da estrutura multiplicativa yjpi ota, ao contar o outro pé, são 3 membros no total.

Os numerais yjpy ota tyytap 'dez' e yjpi ota tyytap 'vinte' foram traduzidos por um informante nativo como "todas as mãos" e "todos os pés”, respectivamente. Contudo, o significado da palavra tyytap, que contém a palavra ty $y$, no dicionário de Landin (1983), significa 'grande'. Essa estrutura ainda precisa ser mais bem explorada, mas, ainda assim, esses numerais apresentam as mesmas bases morfêmicas encontradas nos demais numerais. É interessante notar que a tradução "todos" dada pelos falantes remete ao termo akatyym traduzido como 'todos'. Coutinho-Silva (2008) faz uma análise desse termo que se assemelha a uma quantificação universal. Contudo, esse autor defende que não se trata de quantificação universal, mas de uma sentença subordinada que produz esse efeito de sentido, o que também corrobora a ausência de quantificação nominal nessa língua.

Já o numeral myhint yjpi py 'quinze' parece ser uma irregularidade. Irregularidades são um fato comum em sistemas numéricos, como no Francês, por exemplo, que usa soixante-dix literalmente 'sessenta-dez' para expressar setenta, mas usa quatre-vingt 'quatro vinte' para expressar oitenta. No caso do Karitiana, myhint yjpi py significa 'um pé e mão', o que sugere que a composição aritmética subjacente é $5^{\star} 3$.

Como o Karitiana é uma língua de núcleo final, a estrutura sintática do numeral é uma imagem espelhada da estrutura que Hurford $(1987,2007)$

Revista Letras,

Curitiba, UFPR, n. 96 , pp.546-565, jul./dez. 2017 ISSN 2236-0999 (versão eletrônica) 
postulou para o inglês e da estrutura do Português que foi apresentada acima. A palavra ot, raiz do verbo 'pegar', funciona sintaticamente como uma conjunção coordenativa na formação nos numerais, como mostram as estruturas dos números sypomp yjpi ot 'doze' em (12) e de sypomp yjpi ota ot 'dezessete' em (13) abaixo:
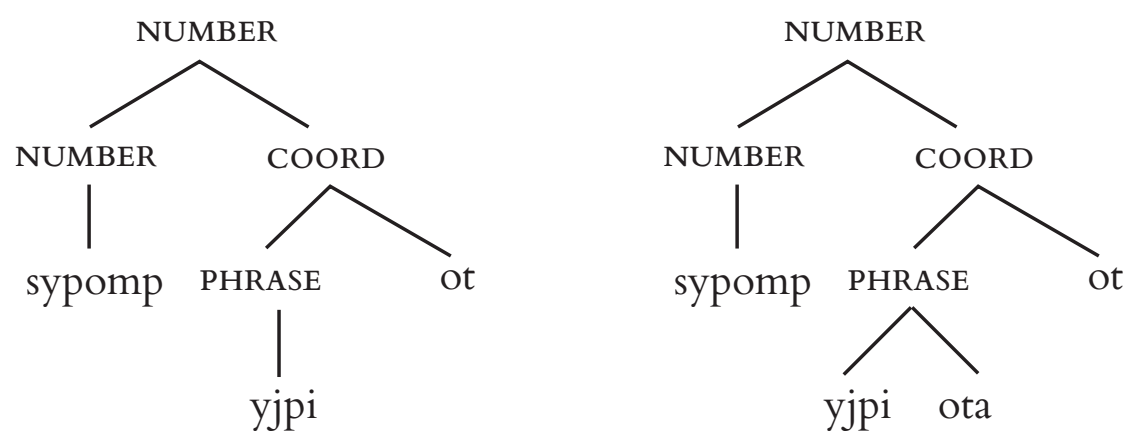

A palavra ot é a marca morfológica da operação de adição nessas estruturas. Ela soma o numeral que está no topo da estrutura com a estrutura multiplicativa. Esta, por sua vez, não apresenta uma marca morfológica. Dentro dessa perspectiva de análise, a palavra ota parece operar como um NUMBER, já que se une com uma base morfêmica sem qualquer partícula como é esperado nas estruturas que expressam a operação de multiplicação.

Abaixo, a estrutura sintática do numeral sypomp yjpy ota ot "sete" é apresentada em (14) e a sua correspondência semântica em (15). É possível observar que os tipos semânticos que Rothstein (2013) propõe funcionam para os numerais Karitiana. Isso é um argumento para o status de base multiplicadora para as palavras yjpy e $y j p i$, e para o status de conjunção coordenativa de ot.

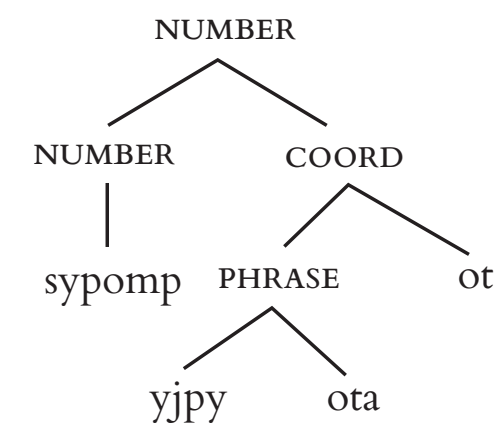

(15)

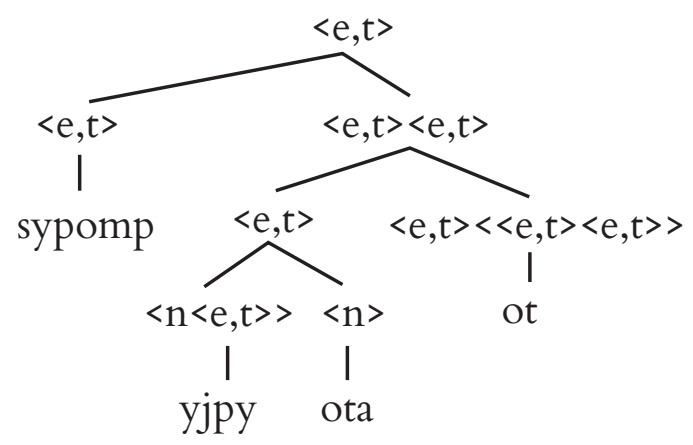

Os tipos semânticos que Rothstein propõe para as palavras utilizadas na formação de numerais compostos, isto é, numerais básicos, conjunções coordenativas e o léxico multiplicador, se aplicam na formação dos numerais compostos do sistema numérico Karitiana. 
J. N.

VIGNADO

A sintaxe e

a semântica

de expressões

numéricas no

Karitiana

Se ota for considerado um NUMBER, é possível derivar a semântica da estrutura interpretada como multiplicação a partir da semântica de suas partes a partir dos tipos semânticos de Rothstein (2013). Na exposição abaixo foi considerado o número 7. O numeral Karitiana para 7 contém a estrutura multiplicativa yjpy ota, o que significa somar ao NUMBER uma outra mão, o que significa pegar 1 outro membro que vale 5 . Nesse caso, ota vale 1 por uma inferência referencial, como se observa abaixo:

$$
\begin{aligned}
& {\left[\left[\operatorname{PHRASE}_{\langle e, t\rangle}\right]\right]=\left[\left[\mathrm{M}_{\langle\mathrm{n}<e, t\rangle}\right\rangle\right]\left(\left[\left[\mathrm{NUMBER}_{\langle\mathrm{n}\rangle}\right]\right]\right) \quad \text { aplicação funcional }} \\
& {\left[\left[\text { PHRASE }_{<e, t}\right]\right]=\left[\left[\text { yjpy }_{\langle\mathrm{n}<e, t\rangle}\right]\right]\left(\left[\left[\operatorname{ota}_{\langle\mathrm{n}\rangle}\right]\right]\right)=\lambda_{\mathrm{n}} \lambda \mathrm{x} .|\mathrm{x}|=5 . \mathrm{n}} \\
& {\left[\left[\text { PHRASE }_{<e, t}\right]\right]=\lambda \mathrm{x} .|\mathrm{x}|=5}
\end{aligned}
$$

O nó sintático COORD é formado pela aplicação da denotação de PHRASE à denotação de ot, como mostrado abaixo:

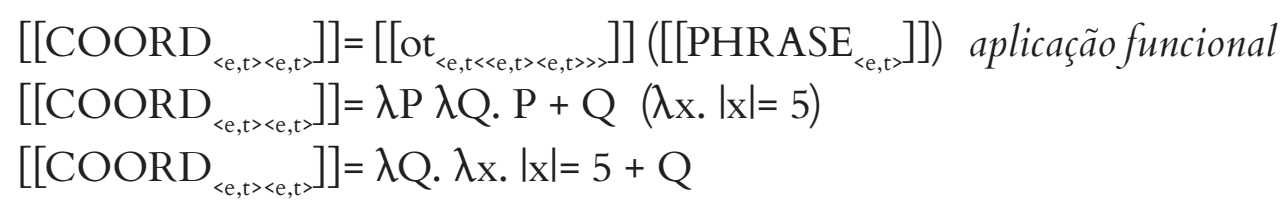

Por fim, a coordenação aplicada a outro numeral compõe a estrutura de adição, como mostrado abaixo:

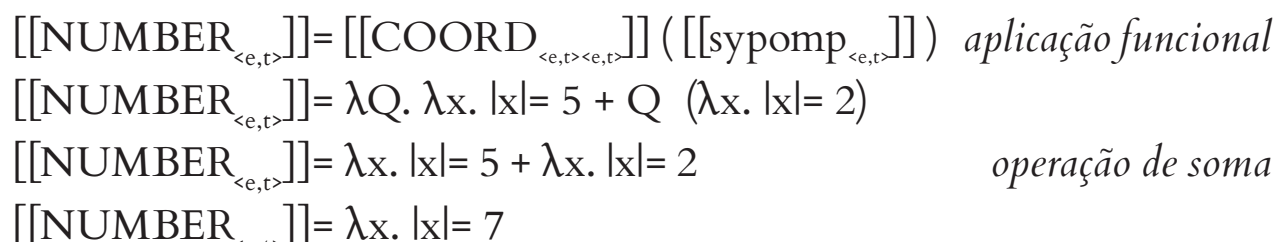

Então, a denotação desse numeral é:

$\left[\left[\right.\right.$ sypomp yjpy ota ot $\left.\left.{ }_{<e, t}\right]\right]=\lambda x .|x|=7$

A exposição da semântica composicional do numeral sypomp yjpy ota ot "sete" em Karitiana mostrou que os tipos semânticos e as denotações que a autora propõe em seu modelo para numerais funcionam em Karitiana se a palavra ota "outro" for analisada como um NUMBER.

Esta análise mostrou que a semântica de ota e seu funcionamento sintático reforçam que esse elemento opera como um NUMBER apesar de não possuir um valor cardinal preestabelecido. Ao indicar o outro membro, seja outra mão ou outro pé, essa palavra permite deduzir quantos são os membros envolvidos na contagem daquele valor numérico. Então, existe algum mecanismo semântico e pragmático a ser mais bem analisado que define a cardinalidade que ota assume

Revista Letras,

Curitiba, UFPR, n. 96 , pp.546-565, jul./dez. 2017 ISSN 2236-0999 (versão eletrônica) 
em uma expressão numérica a partir de sua relação com a base morfêmica multiplicativa.

\section{Considerações finais}

Este artigo apresentou o sistema numérico Karitiana, com seus numerais básicos e seus numerais compostos. A análise foi baseada na sintaxe e na semântica das estruturas numéricas de uma língua indígena brasileira a partir do princípio da estratégia de empacotamento de Hurford (1987), que busca fornecer regras que gerem economicamente e com adequação semântica todas as expressões numéricas bem formadas de uma língua, e da proposta de tipos semânticos para uma semântica composicional de expressões numéricas elaborada por Rothstein (2013).

A análise dos dados mostrou que o sistema numérico Karitiana é de base 5 e que os numerais compostos são formados via bases multiplicadoras e coordenação, como era previsto pela teoria. Até o momento, duas bases morfêmicas multiplicativas foram identificadas na língua: yjpy e yjpi, que, apesar do fato de valerem 5 cada, se distribuem de maneira complementar e hierarquizada.

Assim como outras línguas naturais, o Karitiana apresenta uma marca morfológica nas estruturas interpretadas como adição. Nessa língua, a raiz do verbo "pegar" ot funciona como uma conjunção coordenativa. Em contrapartida, a operação de multiplicação não possui marcação morfológica. Esses fatos

562 linguísticos seguem a tendência dos sistemas numéricos das línguas naturais.

Em síntese, esta análise mostrou que: (i) o sistema numérico Karitiana é de base 5; (ii) a estratégia de empacotamento opera nos numerais Karitiana; (iii) ota é uma expressão vaga que opera semântica e sintaticamente como um NUMBER e cuja cardinalidade é dada por uma inferência referencial baseada nas partes do corpo.

Por fim, a análise contribui para corroborar a hipótese da universalidade da estratégia de empacomento a partir de dados de uma língua não indo-europeia indígena. Os sistemas numéricos são tecnologias cognitivas geradas por nossa faculdade numérica, que, segundo Chomsky (1998), é um subproduto da arquitetura da linguagem natural. Assim, estudar números de línguas não indo-europeias de uma perspectiva formal e gerativa é potencialmente esclarecedor, e pode fazer avançar a compreensão do desenvolvimento da cognição humana e das teorias linguísticas. 
J. N.

VIGNADO

$A$ sintaxe e

a semântica

de expressões

numéricas no

Karitiana

\section{Referências}

ALFORD, M. Developing Facilitative Reading Programmes in Third World Countries, Journal of Multilingual and Multicultural Development, v. 8, n. 6, p. 493-511. 1987.

AMAECHI, M. C. The Syntax of Po Tangle Numerals. Journal of Universal Language, v. 15, n. 2, p. 35-54, 2014.

ANDERSON, L. Vocabulario breve del idioma Ticuna, Revista Peruana de Cultura, v. 8, n. 21, p. 53-68. 1958.

BOSWOOD, J. Quer falar a língua dos Canoeiros? Summer Institute of Linguistics, Brasília, 108 p. 1978.

CHIERCHIA, G. Formal semantics and the grammar of predication. Linguistic Inquiry, v. 16, n. 3, p. 417-443. 1985.

CHOMSKY, N. Language and Problems of Knowledge. Cambridge, MA: MIT Press, 1998

COMRIE, B . Numeral Bases. In: DRYER, M. S.; HASPELMATH, M. (Eds.). The World Atlas of Language Structures Online. Leipzig: Max Planck Institute for Evolutionary Anthropology, 2005. p. 530-533.

CORVER, N.; DOETJES, J.; ZWARTS, J. Linguistic perspectives on numerical expressions: Introduction. Lingua, v. 117, n. 5, p. 751-757. 2007

COUTINHO-SILVA, THIAGO. Aspectos dos sintagmas nominais em karitiana: a quantificação universal. Dissertação de mestrado, Faculdade de Filosofia, Letras e Ciências Humanas-USP, São Paulo, 2008.

EPPS, P.; SALANOVA, A. P. A Linguística Amazônica Hoje. Liames: Línguas Indígenas Americanas, v. 12, p. 7-37, 2012.

FARGETTI, C. M.; SUMAIO, P. A. Numerals in Juruna. Liames: Linguas Indígenas Americanas, v. 15, n. 2, 2015.

FREGE, G. Über Begriff und Gegenstand. In Vierteljahresschrift für wissenschaftliche Philosophie, v. 16, p. 192-205. 1892. Translated as 'Concept and Object' by P. Geach in Translations from the Philosophical Writings of Gottlob Frege, P. Geach and M. Black (eds. and trans.), Oxford: Blackwell, 3ed. 1982.

GREEN, D. Diferenças entre termos numéricos em algumas línguas indígenas do Brasil. Boletim do Museu Paraense Emílio Goeldi-CNPq, Série Antropologia, v. 13, n. 2, p.179-207. 1997.

GRIFFITHS, G. Numerals and demonstratives in Kadiwéu. Arquivos de anatomia e Antropologia . Instituto de Antropologia Professor Souza Marques, v.1, p.6377. 1975.

Revista Letras,

Curitiba, UfPR,

n. 96 , pp.546-565,

jul./dez. 2017

ISSN 2236-0999

(versão eletrônica) 
HURFORD, J. R. The linguistic theory of numerals. Cambridge: Cambridge University Press. 1975.

Language and Number: The Emergence of a Cognitive System. Oxford: Blackwell. 1987.

.The interaction between numerals and nouns. In: PLANK, F. (Ed.). Noun Phrase Structure in the Languages of Europe. Typology of Languages in Europe 7. The Hague: Mouton de Gruyter, 2003. p. 561-629.

A performed practice explains a linguistic universal: Counting gives the Packing Strategy, Lingua, v. 117, p. 773-783, 2007.

IONIN, T.; MATUSHANSKY, O. The Composition of Complex Cardinals. Journal of Semantics, v. 23, n. 4, p. 315-360. 2006.

KARAJÁ, I., FORTUNE, D.; FORTUNE, G. (Eds.) Pré-Matemática para Falantes da Lingua Karajá. Como Transmitimos o Conhecimento Cultural às Nossas Crianças: Como as Crianças Aprendem a Contar e a Calcular. 2a ed. Brasília, Summer Institute of Linguistics, 111 p. 1989.

KAKUMASU, J.; KAKUMASU, K. Dicionário Por Tópicos Urubú-Kaapór -Português. Brasília, Summer Institute of Linguistics, 348 p. 1988.

KOŞANER, Ö. Numerals in Turkish. Open Journal of Modern Linguistics, v. 6, n. 2, p. 131-147. 2016.

LANDIN, D. Dicionário e Léxico Karitiâna - Português. Brasília, Summer Institute of Linguistics. 197 p. 1983.

An Outline of the Syntactic Structure of Karitiana Sentences. Estudos sobre Linguas Tupi do Brasil, Série Linguística 11 SIL, 219-254. 1984.

Dicionário e Léxico Karitiana/Português. Sociedade Internacional de Linguística. Cuiabá-MT, 2005.

LEITE, Y.; FRANCHETO, B. 500 Anos de Línguas Indígenas no Brasil. Ms., 2001.

MULLER, A. ; STORTO, L. ; COUTINHO-SILVA, T. Número e a Distinção Contável-Massivo em Karitiana. Revista da ABRALIN, v. 5, p. 185-213. 2006.

RODRIGUES, A. Linguas brasileiras: para o conhecimento das linguas indígenas. São Paulo: Loyola. 1986.

ROTHSTEIN, S. A Fregean semantics for number words. In: ALONI, M.; FRANKE, M.; ROELOFSEN, F. (Eds.). Proceedings of the 9th Amsterdam Colloquium. 2013. p.179-186. 
J. N. SANCHEZ-MENDES, L. A quantificação adverbial em Karitiana. Dissertação de

VIGNADO

A sintaxe e

a semântica

de expressões

numéricas no

Karitiana Mestrado, Faculdade de Filosofia, Letras e Ciências Humanas-USP, São Paulo, 2009.

STORTO, L. R. Aspects of a Karitiana Grammar. Tese de Doutorado. Massachussetts Institute of Technology. 1999.

STORTO, L.; Rocha, I. Sintaxe e Semântica Verbal em Karitiana. São Paulo: Parábola, 2012.

VIGNADO, J. N. O Sistema Numérico Karitiana e seus Numerais. Relatório de Iniciação Científica, Faculdade de Filosofia, Letras e Ciências Humanas -USP, São Paulo, 2016.

VIVANCO, C. Orações Relativas Em Karitiana: Um Estudo Experimental. Dissertação de Mestrado, Faculdade de Filosofia, Letras e Ciências HumanasUSP, São Paulo, 2014.

WILLIAMS, J. Grammar Notes and Vocabulary of the Language of the Makuchi Indians, S. Gabriel-Modling near Vienna(Austria): International Review Anthropos. 418 p. 1932.

Submetido em: 14-03-2017

Aceito em: 30-06-2017 